\title{
A NOTE ON SOME PARTIALLY TRANSITIVE PROJECTIVE PLANES
}

\author{
D. R. HUGHES
}

1. Introduction. In [3] the author raised the question of the existence of a projective plane of "type $(4, m)$ " with $m=3, n \neq 4$. Using results of Baer and Paige $[1 ; 4]$, we shall show that such a plane does not exist. On the other hand, we will show that a plane of type $(4, m)$ does exist for every $n=p^{2 t}, p$ a prime.

For background material and definitions the reader is referred to [3] and the references given there; in particular, the notion of a planar ternary ring will be important in what follows. However, we briefly define the class of projective planes of type $(4, m)$. Suppose $\pi$ is a projective plane of (finite) order $n$ and $B$ is a collineation group of $\pi$, where $\&$ fixes (element-wise) $m \geqq 3$ points $Q_{i}, i=1,2, \cdots, m$, on a line $K_{0}$, a point $Q_{0}$ not on $K_{0}$, and the lines $K_{0}$ and $K_{i}=Q_{0} Q_{i}$, $i=1,2, \cdots, m$. An ordinary point (line) is a point not on any of the lines $K_{i}$ (a line not containing any of the points $Q_{i}$ ), $i=0,1, \cdots, m$; suppose furthermore that $(B)$ is transitive and regular on the ordinary points and ordinary lines. Then $\pi$ is of type $(4, m)$. It was shown in [3] that if $m>3$, then $n=(m-1)^{2}$; while if $m=3, n \neq 4$, then $n=2^{t}+1$ for some integer $t$ and $(B)$ contains a normal subgroup $\Re_{0}$ which is elementary abelian of exponent two and order $2^{t}$. Furthermore, in the latter case, $\pi$ is $\left(Q_{0}, K_{0}\right)$ transitive with group $\Re_{0}$; this means that $\Re_{0}$ fixes every point on the line $K_{0}$ and every line through the point $Q_{0}$, and that if $P_{1}, P_{2}$ are points of $\pi$, neither one on $K_{0}$, neither one equal to $Q_{0}$, such that $Q_{0} P_{1} P_{2}$ is a line, then $P_{1} x=P_{2}$ for a unique $x$ in $\Re_{0}$. Hence (see $[3 ; 5]$ ) any planar ternary ring for $\pi$ which has $Q_{0}=(0), K_{0}=[\infty,(0,0)]$ will be linear and have its multiplicative loop isomorphic to $\Re_{0}$. (The point (0) is the intersection of the $x$-axis with the line at infinity, and $[\infty,(0,0)]$ is the $y$-axis.)

2. The nonexistence theorem. We begin this section with a theorem due to Paige [4, Theorem IV.2].

THEOREM 1. Let $(R, F)$ be a finite linear planar ternary ring with associative and commutative multiplication, and let $e=\Pi x$, for all nonzero $x$ in $R$. Then $e^{2}=1, x e+x=x+x e=0$ for all $x$ in $R$, and $x e+(x+y)$ $=y$ for all $x, y$ in $R$.

Reccived by the editors November 5, 1956. 
LEMмA 1. Let $(R, F)$ be a finite linear planar ternary ring of odd order, with associative and commutative multiplication. If $R$ possesses more than one element of multiplicative order two, then $x+(x+y)=y$ for all $x, y$ in $R$.

PROOF. If $R$ possesses more than one element of multiplicative order two, then (see [4], for instance) the element $e$ of Theorem 1 is the identity. Hence the lemma follows from Theorem 1.

Now a duality of a projective plane is a one-to-one mapping of points onto lines, lines onto points, which preserves incidence.

THEOREM 2. If $\pi$ is a projective plane coordinatized by a linear planar ternary ring $(R, F)$ with commutative multiplication, in which $x+(x+y)$ $=y$ for all $x, y$ in $R$, then the mapping $T$ below is a duality of $\pi$, of order two:

$$
T:(x, y) \leftrightarrow[x, y] ; \quad T:(m) \leftrightarrow[\infty,(m, 0)] ; \quad T:(\infty) \leftrightarrow L_{\infty} .
$$

Proof. If $(x, y)$ is on $[m, k]$, then $m x+y=k$, so $x m+k=m x+k$ $=m x+(m x+y)=y$; i.e., $(m, k)$ is on $[x, y]$. The rest of the proof is trivial.

In [1], Baer has defined an absolute point of a duality of order two to be a point which is on its image line, and has shown that if $\pi$ is a plane of odd order $n$, with exactly $n+1$ absolute points, then the absolute points form an oval: i.e., a set of $n+1$ points, no three on a line.

THEOREM 3. If $(R, F)$ is a finite linear planar ternary ring of odd order with associative and commutative multiplication, then $R$ possesses exactly one element of multiplicative order two.

Proof. Since the multiplicative group of $R$ has even order, $R$ certainly contains at least one element of multiplicative order two. If there is more than one such element, then by Lemma 1 and Theorem 2 , the plane $\pi$ coordinatized by $(R, F)$ has a duality of order two: it is easy to see that the absolute points of this duality are the $n+1$ points $(\infty)$ and $(0, y)$ for all $y$ in $R$. This set of points is not an oval, so we are done.

CoROllaRY. If $\pi$ is a projective plane of type $(4, m)$ with $m=3$, then $n=4$.

Proof. From the remarks in the introduction, if $n \neq 4$, then $\pi$ possesses a linear planar ternary ring whose multiplicative loop is an elementary abelian group of exponent two; this is impossible, by Theorem 3.

This completes some of the treatment of type $(4, m)$ in [3], since we now can assert that every plane of type $(4, m)$ has order $n=(m-1)^{2}$. 
3. The existence theorem. Suppose $(R, F)$ is a linear planar ternary ring of order $n=(m-1)^{2}$, containing a subset $R_{0}$ of order $m-1$, and suppose $R$ possesses a group $G$ of mappings satisfying:

(a) $(x+y) S=x S+y S$, for all $x, y$ in $R$, all $S$ in $G$;

(b) $(x y) S=(x s) S \cdot y S$, where $s S=1$, for all $x, y$ in $R$;

(c) $(r s) S=r$, where $s S=1$, for all $r$ in $R_{0}$;

(d) if $x, y, u, v$ in $R, x$ not in $R_{0} y, u$ not in $R_{0} v$, then there is a unique $S$ in $G$ such that $x S=u, y S=v$.

Let $\pi$ be the projective plane coordinatized by $(R, F)$; for each $S$ in $G$, define the mapping $S$ of $\pi$ as below (where $s$ is the element $s S=1)$ :

$$
\begin{aligned}
S:(x, y) & \rightarrow(x S, y S), & {[m, k] } & \rightarrow[(m s) S, k S], \\
(m) & \rightarrow((m s) S), & {[\infty,(k, 0)] } & \rightarrow[\infty,(k S, 0)], \\
(\infty) & \rightarrow(\infty), & L_{\infty} & \rightarrow L_{\infty}
\end{aligned}
$$

Using (a) and (b), it is easy to see that the set of all such mappings $S$ form a group (S) (isomorphic to $G$ ) of collineations of $\pi$. From (c) and (d), we deduce that with respect to $(\xi), \pi$ is of type $(4, m)$; the fixed points are $(\infty),(r)$, for all $r$ in $R_{0}$, and $(0,0)$, while the fixed lines are the lines joining these points.

Now we consider the class of left Veblen-Wedderburn systems (V-W systems, for short) which Hall discovered in [2] (note that Hall defines them as right V-W systems). Let $R_{0}$ be a finite field of order $p^{t} \neq 2, p$ a prime, and let $z^{2}-a z-b$ be irreducible over $R_{0}$. Let $R$ be the set of all elements $\lambda x+y$, for $x, y$ in $R_{0}$, where $\lambda$ is some indeterminant; define addition and multiplication in $R$ as follows:

(i) $(\lambda x+y)+(\lambda u+v)=\lambda(x+u)+(y+v)$;

(ii) $y(\lambda u+v)=\lambda(y u)+y v$;

(iii) if $x \neq 0,(\lambda x+y)(\lambda u+v)=\lambda(a u+x v-y u)+y v-x^{-1} u\left(y^{2}-a y-b\right)$. As defined above, $R$ is a linear planar ternary ring with the left distributive law and associative addition; $R$ never possesses the right distributive law, and never has associative multiplication except when $p^{t}=3, a=0, b=-1$ (by the left distributive law, we mean $a(b+c)=a b+a c)$. For further discussion of these and similar systems, see $[2 ; 3 ; 5]$.

For each choice of $d_{1}, d_{2}, e_{1}, e_{2}$ in $R_{0}, d_{1} e_{2}-d_{2} e_{1} \neq 0$, define the mapping $S=S\left(d_{1}, d_{2}, e_{1}, e_{2}\right)$ of $R$ by $(\lambda x+y) S=\lambda\left(d_{1} x+d_{2} y\right)+\left(e_{1} x+e_{2} y\right)$; let $G$ be the set of all such mappings. Then $G$ is a group, and it is easy to see that $G$ satisfies (a) and (c), where the element $s$ defined by $s S=1$ is $s=\lambda\left(-d_{2} D^{-1}\right)+d_{1} D^{-1}, D=d_{1} e_{2}-d_{2} e_{1}$. The verification of (b) is a matter of straightforward but very tedious computation, which we omit. Finally, let us demonstrate (d). 
Let $\lambda x+y, \lambda u+v, \lambda r+q, \lambda w+z$ be elements of $R$. Then $\lambda x+y$ is in $R_{0}(\lambda u+v)$ if and only if $x v=y u$. So we assume $x v \neq y u$, and similarly, $r z \not \neq q w$. Now let $(\lambda x+y) S=\lambda\left(d_{1} x+d_{2} y\right)+\left(e_{1} x+e_{2} y\right)$, etc., where $S$ is yet to be determined. If $(\lambda x+y) S=\lambda r+q,(\lambda u+v) S=\lambda w+z$, we have:

$$
\begin{aligned}
& d_{1} x+d_{2} y=r, \\
& d_{1} u+d_{2} v=w,
\end{aligned}
$$

$$
\begin{aligned}
& e_{1} x+e_{2} y=q . \\
& e_{1} u+e_{2} v=z .
\end{aligned}
$$

But (1) and (2) are uniquely solvable for $d_{1}, d_{2}, e_{1}, e_{2}$, if and only if the determinant $x v-y u$ is not zero; this we have by hypothesis. Furthermore, the solutions will satisfy $d_{1} e_{2}-d_{2} e_{1} \neq 0$ if and only if $r z-q w \neq 0$; this we have also assumed.

So for every $n=p^{2 t} \neq 4, p$ a prime, there is a plane of type $(4, m)$; in [3] an example with $n=4$ was also given. It is of interest that the same class of projective planes was used, with a different collineation group, to give an infinite set of examples of planes of type $(5, m)$. The question of the existence of even one projective plane of type $(6, m)$ is still open, however.

\section{BIBLIOGRAPHY}

1. Reinhold Baer, Polarities in finite projective planes, Bull. Amer. Math. Soc. vol. 52 (1946) pp. 77-93.

2. Marshall Hall, Jr., Projective planes, Trans. Amer. Math. Soc. vol. 54 (1943) pp. 229-277.

3. D. R. Hughes, Partial difference sets, Amer. J. Math. vol. 78 (1956) pp. 650674.

4. L. J. Paige, Neofields, Duke Math. J. vol. 16 (1949) pp. 39-60.

5. Günter Pickert, Projektive Ebenen, Berlin, 1955.

The Ohio State University 